\title{
The ESPRIT Project CreditMine and Its Relevance for the Internet Market
}

\author{
Susanne Köhler ${ }^{1}$, Michael Krieger ${ }^{2}$ \\ ${ }^{1}$ Fraunhofer IAO, Information Engineering, Nobelstr. 12, \\ D-70569 Stuttgart, Germany \\ Susanne.Koehler@iao.fhg.de \\ ${ }^{2}$ MINDLAB Krieger \& Partner, Ingenieure und Informatiker, Marktstr. 31 \\ D-73207 Plochingen, Germany \\ Michael.Krieger@mindlab.de
}

\begin{abstract}
Data mining technology although it is considered as state-of-the-art it is still an embryonic technology employed only by the group of early adopters. Till now very few organisations have made the big investment in bringing together their corporate data in a data warehouse type environment for the purpose of data mining. Only large and wealthy companies have experimented with such methodologies and the outcomes of their efforts were not disclosed, as it was considered confidential material. Thus there is currently a lack of information concerning the effectiveness of such investments. The aim of the project is to provide decision support for companies, especially in the banking sector for investment in data mining.
\end{abstract}

\section{Introduction}

The objective of CreditMine and its internet pendant MarketMine is to facilitate the usage of data mining technology in traditional banking and in internet banking by sensitising for the possibilities of data mining technologies in electronic business. In order to adopt the new technology for such users, examples of successful implementations are needed which will prove the financial and technical feasibility of such investment.

\section{CreditMine}

In the long run, the winners in the competitive financial industry will be those who can gain fractional improvements in understanding and reacting to their customer's needs, by effective customer targeting management. The evaluation of highperformance, analytical and prediction software tools, for the banking industry that can make use of hidden data assets, by employing data mining technologies, within an existed DBMS system is the objective of the project. 
The CreditMine project intends to offer a benchmark study for implementing state-ofthe-art data mining techniques in the banking sector by implementing pilot applications in a medium size bank. The idea is to offer a framework of reference in order to evaluate in advance the feasibility of such investment projects. The goal of the project is to facilitate the usage of data mining technology by less innovative target groups of major business: small and medium size banks all over Europe.

\section{Approach}

Implementation and evaluation of an integrated set of two applications using existing data mining / data warehouse technology and tools:

CustoNeed: to conduct market segmentation by identifying the common characteristics of customers who buy the same products from the bank.

CustoChurn: to predict which customers are likely to leave the company and go to a competitor and whether the return from the customer is higher than the cost to retain him.

Based on the current market needs, CreditMine adapts, enhances, and integrates the latest technologies necessary to design and develop the data mining tools, and evaluates them in real situations faced by a financial institution.

CreditDeploy: a feasibility and evaluation study documents the quantitative and qualitative results of the proposed approach. The lessons learned by the project will be included in the form of guidelines and methodology for setting up similar systems.

\section{MarketMine}

Since there is a strong need in Germany for the use of data mining technologies even for internet Data (e.g. tracking and profiling data from internet banking), MINDLAB has developed a methodology which permits internet market research and internet marketing with the help of data mining technologies and online user interviews in an integrated process chain. This methodology is especially adapted to the needs of internet banks, but it will later also be practicable available for other sectors as well such as online shops etc.

Internet-sites provide a multitude of data caused by the comprehensible use of these sites. User actions and time spent by the user on a certain internet site can be registered and analysed in detail which allows to define exact profiles of customer habits. By using tracking data, the newly developed method makes it possible to register user behaviour and to segment these data in groups with the same or a similar behaviour. The developed methodology considers the strict European provisions of data privacy.

The objective of this research project which strongly involves the practical side, is to integrate data mining applications used in internet market research into the marketing process which is to be followed. In this manner, possible synergies can be made use of. 